\title{
Impact of dimensionality and confinement on the electronic properties of mercury chalcogenide nanocrystals
}

Charlie Gréboval ${ }^{1}$, Eva Izquierdo², Clément Livache ${ }^{1,2}$, Bertille Martinez ${ }^{1,2}$, Marion Dufour ${ }^{2}$, Nicolas Goubet $^{1,2}$, Nicolas Moghaddam², Junling Qu${ }^{1}$, Audrey Chu ${ }^{1}$, Julien Ramade ${ }^{1}$, Hervé Aubin², Hervé Cruguel $^{1}$, Mathieu Silly ${ }^{3}$, Emmanuel Lhuillier ${ }^{1 *}$, Sandrine Ithurria ${ }^{2}$

1Sorbonne Université, CNRS, Institut des NanoSciences de Paris, INSP, F-75005 Paris, France

'Laboratoire de Physique et d'Etude des Matériaux, ESPCI-Paris PSL Research University, Sorbonne Université Univ Paris 06, CNRS, 10 rue Vauquelin 75005 Paris, France.

${ }^{3}$ Synchrotron-SOLEIL, Saint-Aubin, BP48, F91192 Gif sur Yvette Cedex, France

\begin{abstract}
We demonstrate the growth of 2D nanoplatelets (NPLs) made of a HgTe/CdS heterostructure, with an optical absorption reaching the shortwave infrared range. The material is an interesting platform to investigate the effect of dimensionality (OD vs 2D) and confinement on the electronic spectrum and carrier dynamics in colloidal materials. We bring consistent evidence for the $p$-type nature of this material from transport and photoemission measurements. The majority carrier dynamics probed using pump-probe photoemission is found to be mostly dependent on the presence of a confinement barrier at the surface rather than on the material dimensionality. The minority carrier, on the other hand, is strongly affected by the material shape showing longer lived minority carrier in 2D NPLs compared to their OD equivalent with similar band gap. Finally, we test the potential of this material for photodetection in the short-wave infrared range (SWIR) and show that fast photoresponse and detectivity reaching $10^{9}$ Jones at room temperature can be achieved.
\end{abstract}

Keywords: nanoplatelets, HgTe, confinement, dimensionality, carrier dynamics, infrared

To whom correspondence should be sent: el@insp.upmc.fr

Table of content graphic

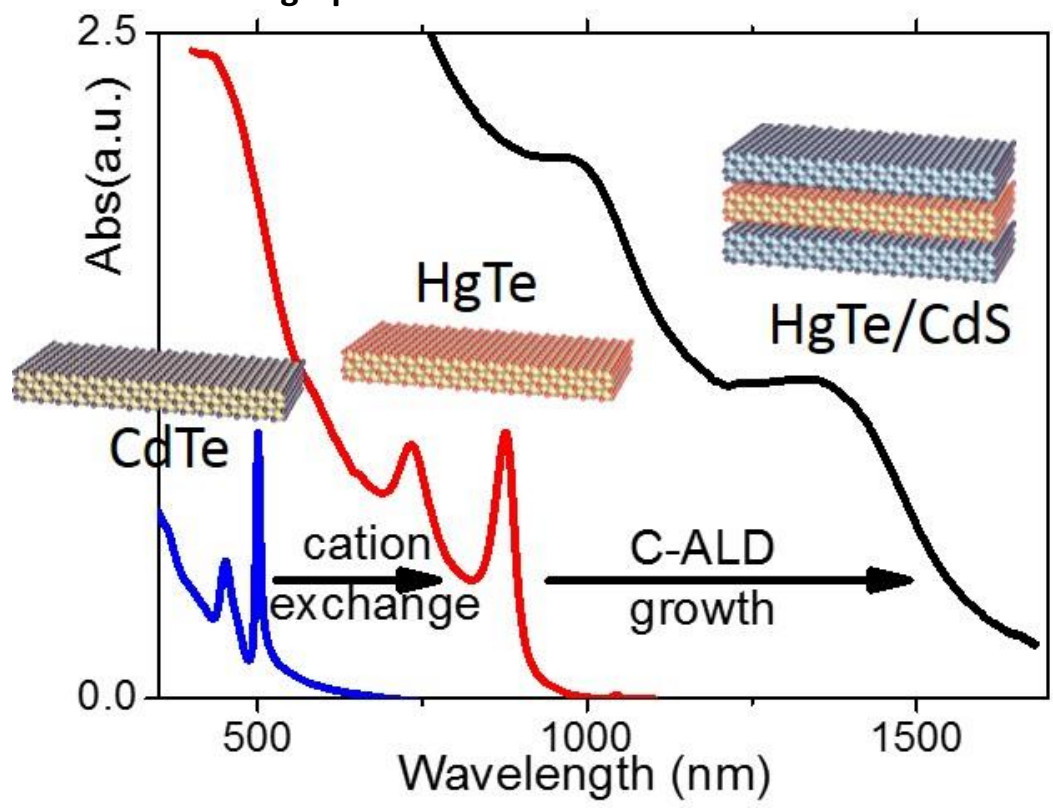

Infrared spectra of CdTe, HgTe and $\mathrm{HgTe} / \mathrm{CdS}$ nanoplatelets 


\section{INTRODUCTION}

Among colloidal quantum dots (CQDs), 2D nanoplatelets ${ }^{1,2}$ (NPLs) of II-VI semiconductors present unique optical properties with an exceptionally narrow photoluminescence (PL) signal. ${ }^{3}$ In the visible range, this makes CdSe NPLs highly promising for the design of wide gamut display. This property results from their specific growth mechanism ${ }^{4-6}$ which induces no roughness along the only confined direction. However, this growth mechanism was up to recently limited to Cadmium chalcogenides with optical features in the visible range of wavelengths.

Imaging in the short-wave infrared range of wavelengths (between 1 and $3 \mu \mathrm{m}$, SWIR) is useful in many applications such as active imaging, bio-imaging, telecom or LIDAR detection. To achieve absorption in the SWIR, narrow band gap materials ${ }^{7,8}$ have to be used. PbS nanosheets ${ }^{9,10}$ have been synthetized ${ }^{11,12}$ and show tunable absorption ${ }^{13}$ almost up to the bulk band gap value. ${ }^{14}$ They also present a large lateral extension ${ }^{15-17}$ (up to several $\mu \mathrm{m}$ ) which is of great interest for the design of single-particle devices such as field effect transistors ${ }^{18,19}$ (FETs) and photodetectors. ${ }^{9}$ On the other hand, $\mathrm{PbS}$ nanosheets remain quite strongly aggregated and their optical features are broad due to scattering and lack of atomic scale control of the thickness. In this sense, 2D colloidal materials with well-defined IR optical features were lacking up to the recent synthesis of HgX NPLs by Izquierdo et al. ${ }^{20}$ These NPLS were obtained from a cation exchange on cadmium chalcogenides NPLs.

Mercury chalcogenides, especially HgTe and HgSe, are interesting candidates for SWIR detection because they are semimetals under bulk form. Consequently, band edge energy of $\mathrm{HgTe}$ or $\mathrm{HgSe}$ nanocrystals is only due to confinement and can theoretically be as low as wanted ${ }^{21}$. The HgTe NPLs obtained by cation exchange show narrow absorption and $P L$ in the near infrared (IR) range (around $900 \mathrm{~nm}$ ). The cation exchange is nevertheless self-limited, meaning that only alloys ${ }^{22,23}$ have been obtained in the case of quantum dots and only surface exchange over the first two monolayers is achieved for NPLs ${ }^{24}$. In practice attempts to conduct the process on NPLs thicker than 3 monolayers have led to CdXHgX core-shell heterostructures. ${ }^{24}$ The exchange is limited to the two external planes of the NPLs. These 2D heterostructures based on $\mathrm{HgX}$ were not able to reach the SWIR range.

To keep the advantages of a 2D geometry while pushing the band edge energy in the SWIR range, one has to find a way to decrease the confinement along the thickness of HgTe nanoplatelets. In this paper, we describe the growth of a CdS shell on HgTe nanoplatelets to obtain tunable optical features in the SWIR range. Beyond the possibility to push the absorption toward redder wavelengths, the promise of such heterostructures is also to reduce the exciton binding energy. In 2D NPLs, the binding energy ${ }^{25,26}$ is larger than their OD counterpart (HgTe 2D nanoplatelets are certainly the most confined materials with a confinement energy around $1.5 \mathrm{eV}$ ), which prevents the effective splitting of the electron-hole pair. ${ }^{27}$ Building a heterostructure has previously been demonstrated as a viable way to enhance charge dissociation. ${ }^{28}$ This is why in the following, we investigate the electronic properties of the $\mathrm{HgTe} / \mathrm{CdS}$ heterostructure. More specifically, we focus on the spectrum of this material in absolute energy scale which is critical for the future integration of the material into devices. We then compare the carrier relaxation dynamics of majority and minority carriers of HgTe NPLs (2D material), HgTe/CdS NPLs (2D material + confinement) and HgTe CQDs (OD material with same band gap as the HgTe/CdS NPLs) to get the influence of dimensionality and confinement on transport. To do so, we use a combination of wide bandwidth transient photocurrent measurements, pump-probe photoemission and transport measurements conducted in a FET configuration. Finally, we investigate the photoconductive properties of the HgTe/CdS NPLs and discuss how the observed changes in carrier dynamics impact their detection performances.

\section{DISCUSSION}

This paper is focused on the optoelectronic properties of HgTe/CdS NPLs because they combine a 2D aspect with a reduced confinement. The growth of HgTe/CdS NPLs relies on three steps, see Figure 1a: (i) growth of CdTe NPLs using the procedure described by Pedetti et al, ${ }^{29}$ (ii) cation exchange to replace cadmium atoms by mercury ones, according to the procedure of Izquierdo et al. ${ }^{20}$ And (iii) the CdS shell growth by colloidal atomic layer deposition (c-ALD) ${ }^{30}$. 
The synthetized core NPLs present three monolayers in thickness (ie there are 3 plans of Te surrounded by 4 plans of cations). This corresponds to a thickness of $1.1 \mathrm{~nm}$, while the lateral extension is typically of $100 \mathrm{~nm} \times 200 \mathrm{~nm}$. The shape of the particles is preserved during these steps as confirmed by electron microscopy, see Figure $1 \mathrm{~b}$ and $\mathrm{c}$. The initial CdTe NPL spectrum presents a very narrow absorption feature at $500 \mathrm{~nm}$, see Figure 1e. After the cation exchange the absorption edge is strongly redshifted and appears at $870 \mathrm{~nm}$.
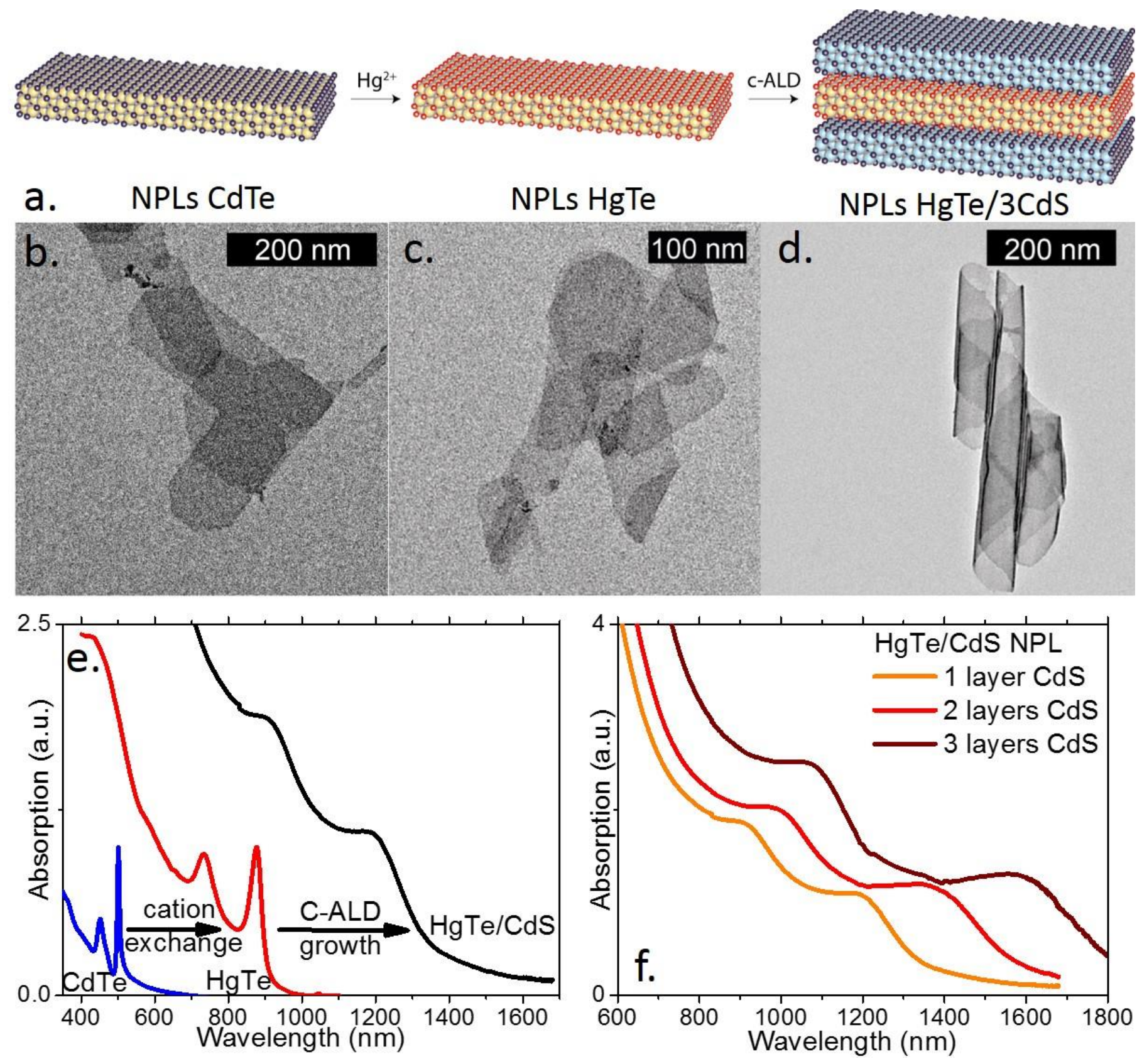

Figure 1 a. Scheme of the growth mechanism used to achieve HgTe/CdS core-shell NPLs. b. TEM image of CdTe NPLs. c. TEM image of HgTe NPLs. d. TEM image of HgTe/3CdS NPLs. e. Absorption spectrum of CdTe, HgTe and HgTe/CdS (1 layer of CdS) NPLs. f. Absorption spectrum of HgTe/CdS NPLs with 1, 2 and 3 layers of CdS in the shell.

The growth of the CdS shell is conducted using the room temperature c-ALD procedure. ${ }^{30}$ The method relies on the successive exposition of the NPLs to cation and anion precursors. The most conventional method is to phase-transfer the particles from a non-polar solvent to a polar solvent ( $\mathrm{N}$-Methyl Formamide typically) and conducts the growth in this polar phase. However, this approach has been unsuccessful (ie no shift of the excitonic feature, nor evidence of $\mathrm{Cd}$ deposition in energy dispersive $\mathrm{X}$-ray spectroscopy). We thus choose to conduct the C-ALD while keeping the material into the non-polar phase, see experimental section for details. The $2 \mathrm{D}$ shape of the material is preserved, but the particles are enrolled due to the lattice mismatch ( $\approx 10 \%)$ between HgTe and CdS, see Figure $1 \mathrm{~d}$, S1 and S2 form TEM imaging. While the diffraction pattern, see figure $S 3$ and table $S 1$, is almost unaffected by the cation exchange 
step, because CdTe and HgTe have very close lattice parameter, we observe a strong modification of the peak relative to HgTe after the growth of the CdS shell. In particular, we can notice a contraction of the lattice parameter of $5 \%$. After the growth of the CdS shell, the absorption is again strongly redshifted and appears at $1200 \mathrm{~nm}$ with 1 layer of CdS, see Figure 1e. As a thicker (3ML) CdS shell is grown, the absorption is further redshifted and the band edge reaches $1600 \mathrm{~nm}$. This shift is the combination of reduced confinement and strain ${ }^{31}$. This is the first time that $\mathrm{HgTe}$ based 2D NPLs reach absorption in the SWIR range. In the following, we will describe a HgTe NPL with $n$ layers of CdS shell as $\mathrm{HgTe} / \mathrm{nCdS}$. We will more specifically focus on $\mathrm{HgTe} / 3 \mathrm{CdS}$ since it has a similar band gap energy as InGaAs semiconductor which is the current leading technology for photodetection in the SWIR range.

Table 1 Atomic ratios of S, Cd, Te and $\mathrm{Hg}$ in $\mathrm{HgTe} / 1 \mathrm{CdS}$ measured by energy dispersive $\mathrm{X}$-ray spectroscopy (EDS) and expected from the number of layers in the NPL.

\begin{tabular}{lllll}
\hline \hline HgTe/1CdS & S (\%) & Cd (\%) & Te (\%) & Hg (\%) \\
\hline Determined by EDS & $16 \pm 2$ & $16 \pm 2$ & $29 \pm 2$ & $39 \pm 2$ \\
\hline $\begin{array}{l}\text { Predicted } \\
\text { stoichiometry }\end{array}$ & 18 & 18 & 27 & 36 \\
\hline \hline
\end{tabular}

Table 2 Atomic ratios of S, Cd, Te and $\mathrm{Hg}$ in $\mathrm{HgTe} / 3 \mathrm{CdS}$ measured by energy dispersive $\mathrm{X}$-ray spectroscopy (EDS) and expected from the number of layers in the NPL.

\begin{tabular}{lllll}
\hline \hline HgTe/3CdS & S (\%) & Cd (\%) & Te (\%) & Hg (\%) \\
\hline Determined by EDS & $30.5 \pm 2$ & $30.5 \pm 2$ & $18 \pm 2$ & $21 \pm 2$ \\
\hline $\begin{array}{l}\text { Predicted } \\
\text { stoichiometry }\end{array}$ & 32 & 32 & 16 & 21 \\
\hline
\end{tabular}

Beyond the optical shift, the growth of the CdS shell can be confirmed by energy dispersive X-ray spectroscopy. Table 1 and Table 2 confirm that the CdS material is incorporated in the heterostructure in a very similar amount as the one expected for infinite layers. In addition, photoemission measurements, conducted on the Tempo beamline of Soleil synchrotron, further confirm the presence of $\mathrm{Cd}$ and $\mathrm{S}$ in the final material, see Figure $2 \mathrm{a}$. A detailed analysis of the core levels is given in Figure S5. The electronic spectrum of the material is reconstructed using a combination of photoemission and infrared spectroscopy. Photoemission is used to locate valence band and vacuum level with respect to the Fermi level which reveals the doping and work function of the material, see Figure $2 \mathrm{~b}$. IR spectroscopy is used to determine both the band edge energy and the conduction band energy. The work function of the material is found to be $4.7 \pm 0.1 \mathrm{eV}$, see Figure $2 \mathrm{c}$, which is consistent with previous observation on HgTe nanocrystals ${ }^{32}$.

The energy profile of the HgTe/3 CdS NPLs is compared with the one obtained for pure HgTe NPLs (with a larger confinement) and with HgTe CQDs (typical size $6 \mathrm{~nm}$ ) with similar band gap in order to understand the effect of material dimensionality, see Figure $2 \mathrm{c}$ and $\mathrm{d}$. Because $\mathrm{CdS}$ has a wide band gap (2.4 eV), it forms with HgTe a type I heterostructure ${ }^{33}$. Nevertheless the (quantum and dielectric) confinement is so strong in the HgTe NPLs that the wavefunction partly delocalized in the CdS shell, leading to the observed reduction of band edge energy. This is consistent with the band edge shift observed on HgTe NPLs as the surface chemistry is modified ${ }^{20}$. In the following, we will study how this barrier not only affects the spectrum but also the carrier dynamics. Small size HgTe CQDs can be grown to have a similar band gap as the HgTe/3CdS NPLs, in this case the energy profile is found to be very similar, see Figure $2 \mathrm{c}$. Those two materials will be compared to understand the effect of the CdS confinement. 

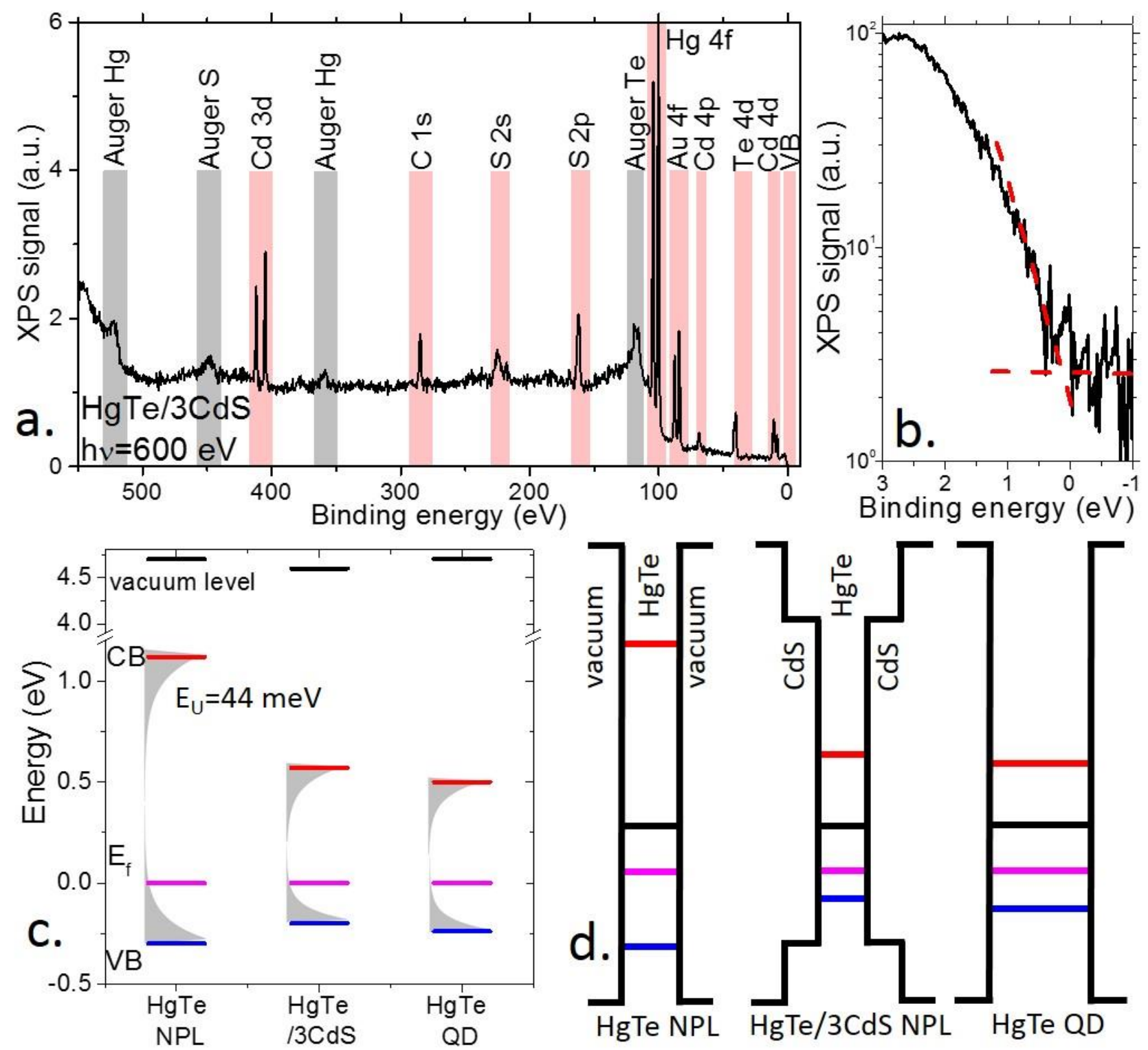

Figure 2 a. X-ray photoemission overview spectrum from HgTe/3CdS NPLs. Photon energy is set at $600 \mathrm{eV}$. Red lines relate to core level peaks, while grey lines refer to Auger features. b. Signal relative to the valence band in the $X$-ray photoemission spectrum from $\mathrm{HgTe} / 3 \mathrm{CdS}$ NPLs. c. Electronic spectrum in absolute energy scale for HgTe NPLs, HgTe/3CdS NPLs and HgTe QDs (6 nm). The Fermi level has been set equal to zero (pink). Valence band position is extracted from the low binding energy part of the photoemission spectrum, while the conduction band energy is determined with respect to the valence band using the absorption band gap. Finally the vacuum level is determined from the cut-off of the secondary electrons. The grey levels in the gap correspond to trap states which distribution width can be determined using transient photocurrent measurements and was measured to be around $44 \mathrm{meV}$. A more detailed description of these measurements is provided further in the article, especially on Figure 5. $d$. Scheme of the valence band and conduction band energy profile for the Hgte NPLs, the HgTe 3CdS NPLs and the HgTe QDs.

The relative position of the Fermi level with respect to the middle of the band gap is used to determine the nature of doping in the material. In all cases, the Fermi level is found to be closer to the valence band suggesting a $p$-type character for the material, see Figure 2c. Again this is consistent with previous observation on HgTe where a switch from $p$-type to $n$-type has been observed with the reduction of confinement energy. ${ }^{21,26} \mathrm{The}$ HgTe NPLs and QDs being strongly confined (> $700 \mathrm{meV}$ of confinement), we expect them to be $\mathrm{p}$-type. The nature of the majority carrier is also confirmed by transport measurements. In this case, a thin film of NPLs or QDs is prepared and crosslinked using ethanedithiol as coupling ligand to increase the inter-nanoparticle electronic coupling. A mixture of $\mathrm{LiClO}_{4}$ in polyethylene glycol is used as ion-gel electrolyte ${ }^{34}$, see Figure S7. This electrolyte gate combines several advantages such as a large capacitance, air operability, low bias operability and possibility to gate thick films thanks to the diffusion of ions within the nanocrystal film. The transfer curves are given in Figure 3a-c and present a current modulation 
(on/off ratio) above two orders of magnitude. HgTe NPLs with and without shells as well as HgTe CQDs exhibit a clear increase of the conductance under hole injection (ie negative gate bias), while the electron conduction remains limited. The temperature dependency of the transport has been investigated, see Figure $3 \mathrm{~d}$-f. Close to room temperature, the I-T curve is well fitted by an Arrhenius law. The extracted activation energy, which quantifies the impact of temperature on the device dark current, is typically $250 \mathrm{meV}$ for the HgTe NPLs. HgTe/3CdS and the HgTe CQDs present a lower activation energy of 140-150 meV. This value is quite close to the measured energy spacing between the valence band and the Fermi level and thus reflects the thermal activation of holes up to the valence band.
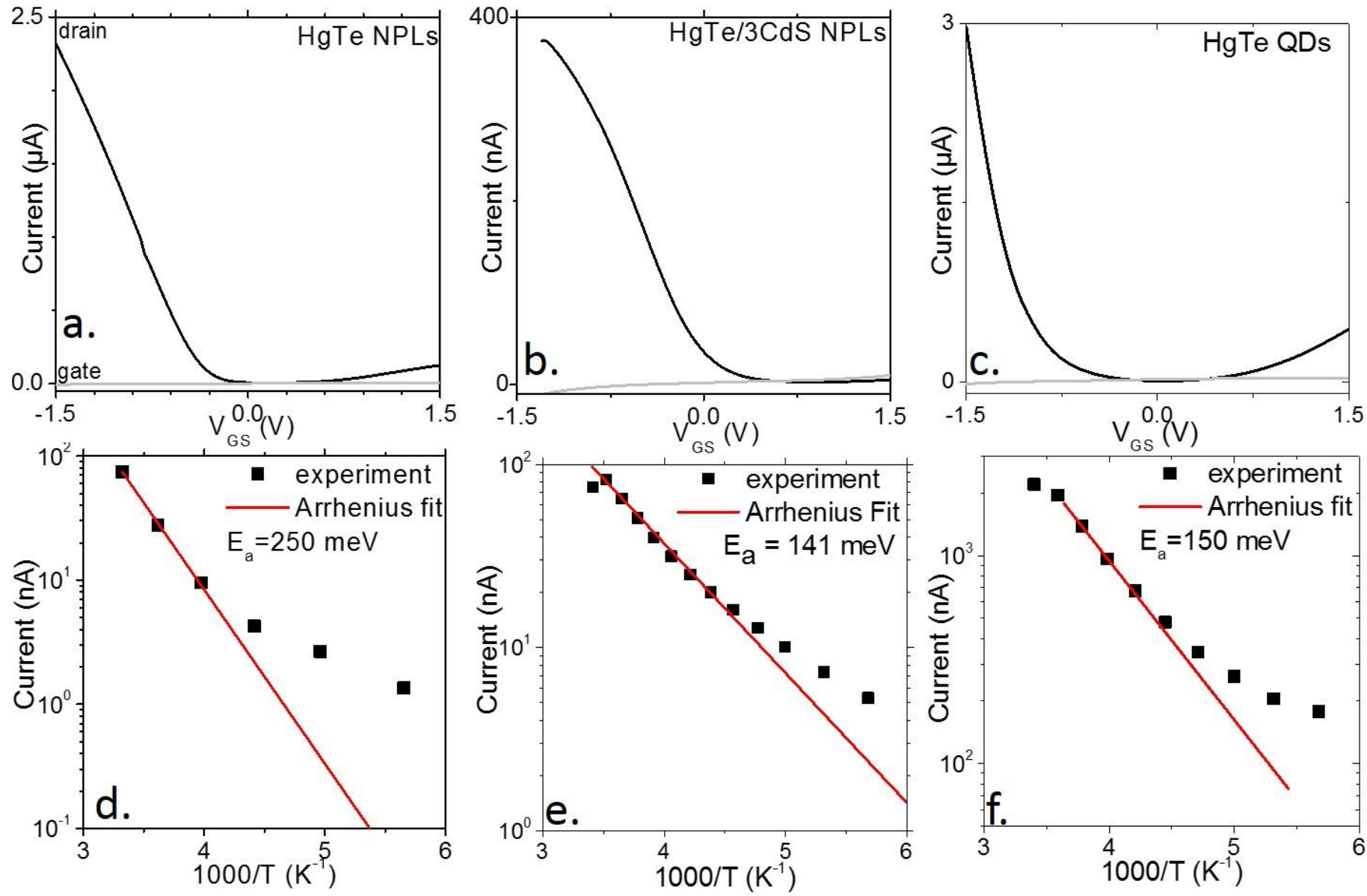

Figure 3 Transfer curves (drain and gate currents as a function of applied gate bias) for thin films of HgTe NPLs (a), $\mathrm{HgTe} / 3 \mathrm{CdS}$ NPLs (b) and HgTe QDs (c). The applied drain-source bias is $500 \mathrm{mV}$ for HgTe and $100 \mathrm{mV}$ for HgTe/CdS. Current as a function of temperature for thin films of HgTe NPLs (d), HgTe/3CdS NPLs (e) and HgTe QDs (f). For the I-T curve, the measurement is conducted in a two contact geometry (ie without gate)

We finally use a third strategy to investigate the nature of the majority carriers in this confined form of HgTe. To do so, we have used transient photovoltage measurements conducted in a pump-probe configuration. ${ }^{35-37} \mathrm{~A}$ scheme of the setup is given in Figure 4a. A short ( $80 \mathrm{fs})$ pulse of light $(\lambda=800 \mathrm{~nm})$ is generated from a Ti:Sapphire laser and shone on the sample. After this pump excitation, a $600 \mathrm{eV}$ photon source from the synchrotron is used to probe every $10 \mathrm{~ns}$ the photoemission signal from the $\mathrm{Hg} 4 \mathrm{f}$ state. The binding energy is then plotted as a function of time and the sign of the observed energy shift is a signature of the majority carrier nature. More details about the principle of this measurement can be found in the supporting information, see figure $\mathrm{S6}$.

In all cases, we have observed a negative shift (ie a decrease of the binding energy) which is consistent with a p-type semiconductor. The decay time of the binding energy is related to the majority carrier diffusion time. ${ }^{32}$ The hole lifetime is found to be 230-240 ns for HgTe NPLs without CdS barrier and for HgTe QDs, see Figure 4b. It thus poorly depends on band gap value nor dimensionality. On the other hand, the introduction of the CdS shell considerably increases the hole lifetime up to $\approx 1 \mu \mathrm{s}$. CdS presents a type I band alignment with the HgTe semimetal. CdS forms a barrier in addition to the existing one resulting from the ligand, which slows down the hopping transfer from one nanoparticle to another. As a result, we can conclude that the dynamics of majority carriers is driven by the confining barrier. 

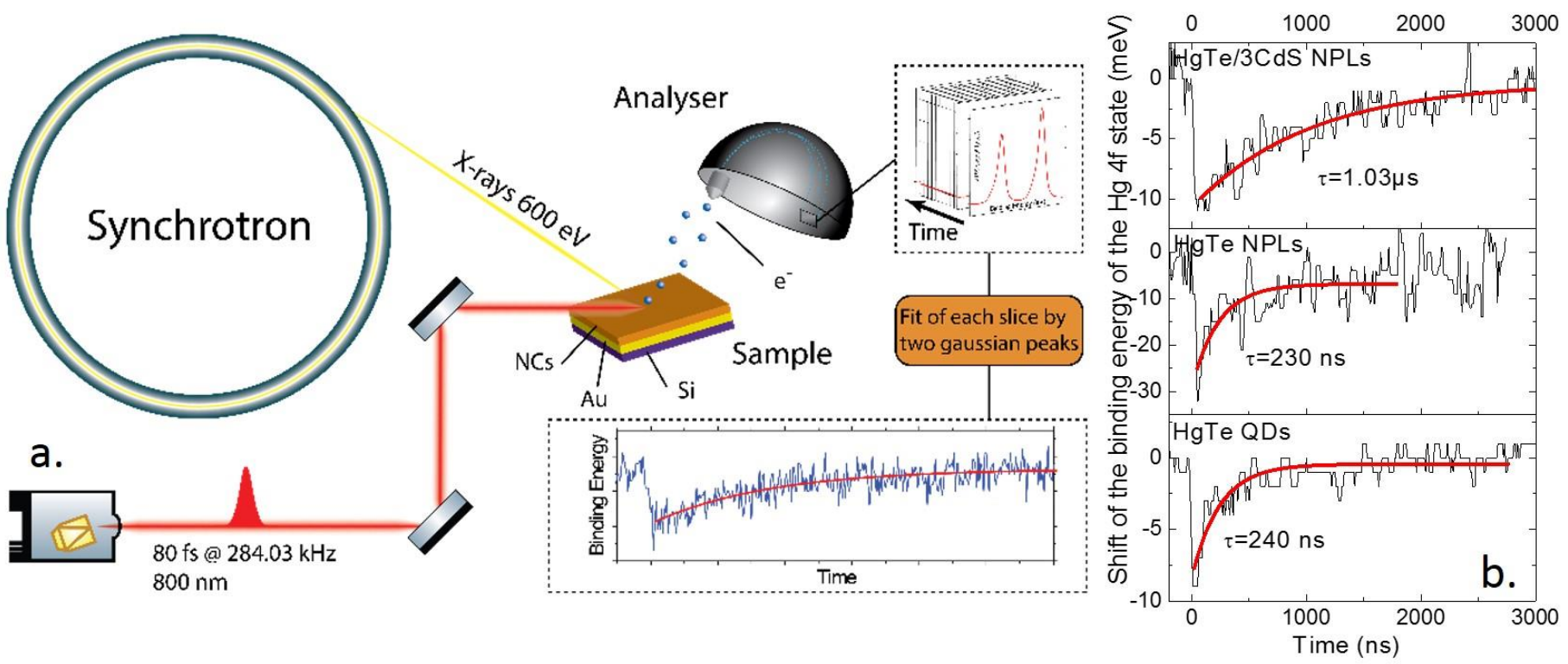

Figure 4 a. Scheme of the pump probe experiment used to measure the majority carrier lifetime. Typically, an $800 \mathrm{~nm}$ pulsed laser is used to excite the system. Then, every $10 \mathrm{~ns}$, a photoemission spectrum of the $\mathrm{Hg} 4 \mathrm{f}$ state is acquired. The peak position is then fitted and plotted as a function of time. $b$. Shift of the binding energy relative to the $\mathrm{Hg} 4 \mathrm{f}$ state as a function of time after illumination by an 80 fs long pulse of $800 \mathrm{~nm}$ light for thin film of HgTe NPLs, HgTe/3CdS NPLs and HgTe QDs.

We also investigated the dynamics of the minority carriers (ie the electrons). Here we used a wide dynamic setup ${ }^{38}$ which combines both broad temporal bandwidth (from ns to $10 \mathrm{~ms}$ ) and large signal dynamics (4 orders of magnitude typically). A scheme of this transient photocurrent experiment is given as an inset of Figure 5a. Briefly, a short (0.9 ns) UV $(\lambda=355 \mathrm{~nm})$ pulse is shone on a thin film of nanoparticles deposited on biased interdigitated electrodes. The wide dynamic of the setup reveals a long living photoresponse, see Figure 5a. As discussed previously by Martinez et al, ${ }^{21}$ the temporal response can be split in two parts. At early time scale, there is a fast decay corresponding to carrier recombination, this decay time generally matches well with the PL decay time. ${ }^{39}$ In the case of HgTe CQDs, we observe a fast decay of $0.5 \mathrm{~ns}$, see Figure $5 \mathrm{~b}$. This measurement is actually limited by the pulse length which is consistent with previously reported PL decay ${ }^{40}$ measured on similar size of HgTe CQDs for which a bi-exponential behavior was observed with decay time of 50 ps and 2 ns. On the other hand, the decay time measured for the HgTe and the $\mathrm{HgTe} / 3 \mathrm{CdS}$ NPLs is longer than the setup resolution and has been determined to be $1.8 \mathrm{~ns}$. This result is quite promising for the integration of such NPLs for photodetection since this decay time will drive the magnitude of the photoresponse.

At long time scale, the photocurrent follows a power law, see Figure $5 c$, which is the signature of multi-trapping transport. ${ }^{41,42}$ In this regime, charges which have been trapped at early times are thermally released at the band edge. The deeper the trap, the longer it takes to be released. The exponent of the power law fit ( $b \approx 0.38$, on Figure $5 c$ ) directly relates to the Urbach energy $\left(E_{U}\right)$ which describes the in-gap distribution of traps, through the relation $b=-1+\frac{k_{b} T}{E_{U}}$ with $\mathrm{k}_{\mathrm{b}} \mathrm{T}$ the thermal energy. While the value of the Urbach energy has been found to be strongly dependent on the nanocrystal surface chemistry, ${ }^{21}$ it appears that particle shape and presence of barrier barely affects the trap distribution. We measured a value of $44 \pm 3 \mathrm{meV}$ for all materials, see Figure $5 \mathrm{c}$. 

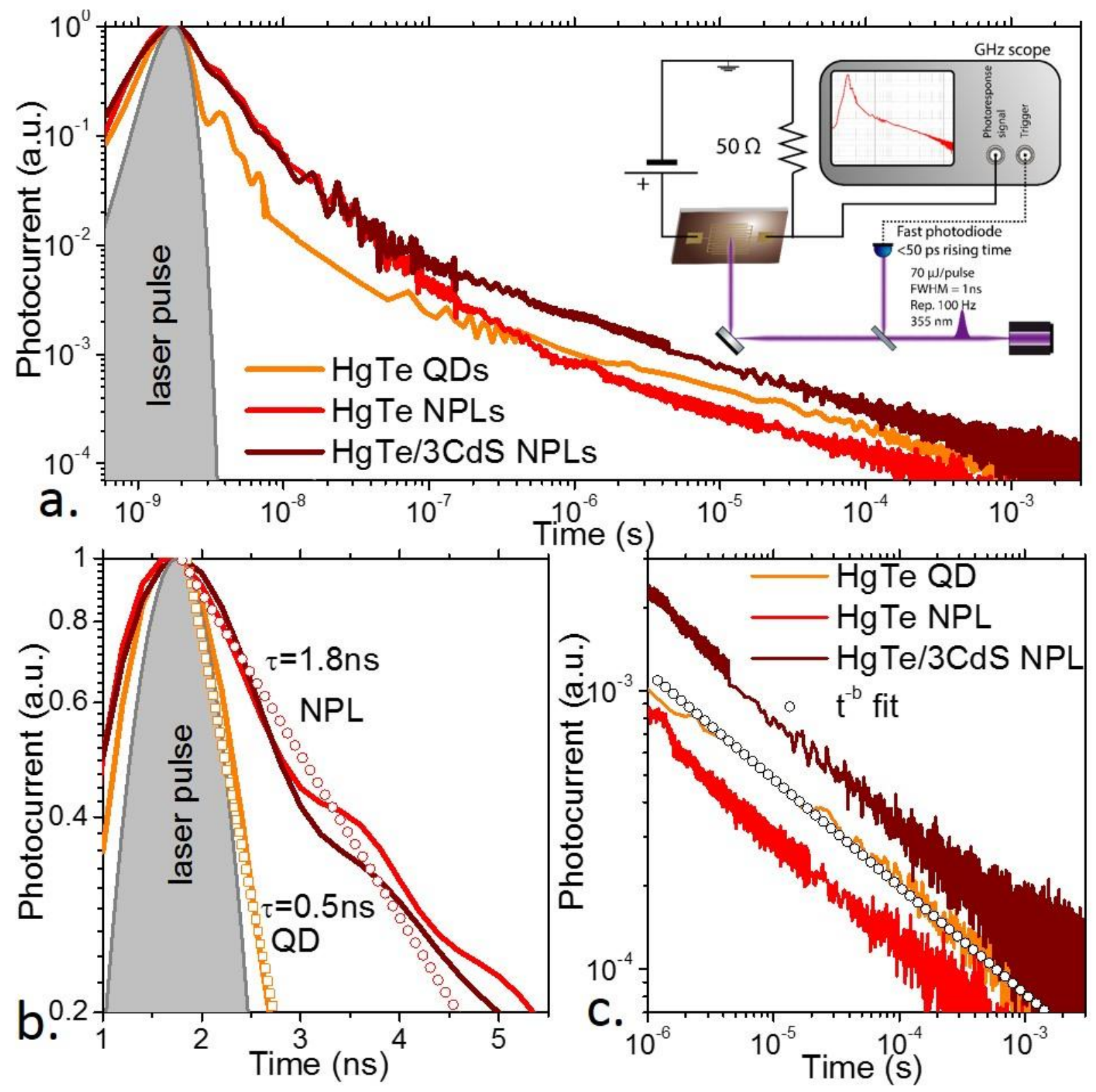

Figure 5 a. Photocurrent as a function of time after illumination by a $0.8 \mathrm{~ns}$ long pulse of $355 \mathrm{~nm}$ light. The inset is a scheme of the setup used for measurement. $b$. Zoom on the graph of part a. at early timescale. The scattered lines are exponential fit. The associated decay time are also provided. c. Zoom on the graph of part a. at long timescale.

To conclude, we have investigated the potential of these HgTe/3CdS NPLs for detection in the SWIR. First, we have measured the photocurrent spectrum and compared it with the absorption of the nanoparticles before ligand exchange. As often, with thiol ligand exchange, the optical feature gets broader. ${ }^{43}$ More surprisingly, we have observed a blue shift of the transition, see Figure 6a. This suggests that the NPL surface is partly etched as ligand exchange is conducted and that thiols strip some of the surface cadmium. The material presents a clear photoresponse under excitation by a laser source at $1.55 \mu \mathrm{m}$, see Figure $6 \mathrm{~b}$, with a strong increase of the conductance under illumination. The responsivity has been determined and is typically in the few $100 \mathrm{~s} \mu \mathrm{A} / \mathrm{W}$ under illumination in the $\mathrm{mW}$ range, see Figure $6 c .{ }^{44,45}$ The device also presents a fast time response, see Figure $6 \mathrm{~d}$ and S3, with a rise and fall time below $10 \mu \mathrm{s}$ which actually is limited by the laser rise time in this experiment. The noise (see Figure S9) has been determined to be $1 / f$ limited, see the inset of Figure $6 \mathrm{e}$. This leads to device detectivity reaching $10^{9}$ Jones at room temperature and under low flux, see Figure 6e. Performance-wise the material is not reaching state of the art device made of $\mathrm{PbS}$ and operated at the same wavelength. ${ }^{46}$ This difference results from the much larger dielectric constant of $\mathrm{PbS}^{47}\left(\varepsilon_{\mathrm{Pbs}}=20\right.$ 40) compared to $\mathrm{HgTe}^{48}\left(\varepsilon_{\mathrm{HgTe}}=13\right)$ : as a result, charges behave as quasi-free carriers in PbS while the exciton remains strongly bound in HgTe. In addition, the 2D aspect of the NPLs further enhanced the exciton binding energy. ${ }^{26}$ It was 
nevertheless proposed that such difficulty can be overcome by integrating the NPL into nanotrench device ${ }^{27}$ where the high exciton binding energy can be balanced by the application of a high electric field.
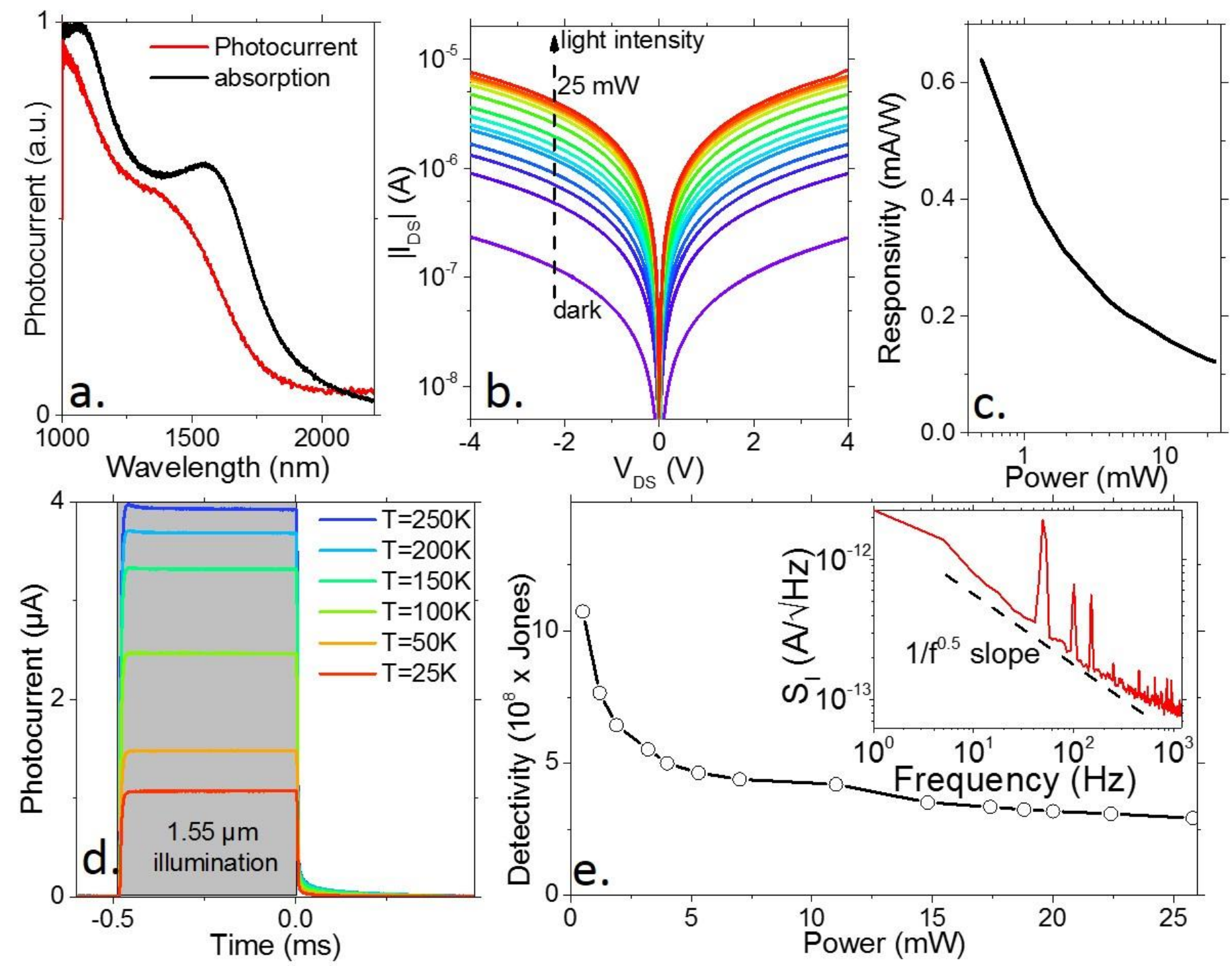

Figure 6a. Photocurrent and absorption spectra for a thin film of HgTe/3CdS NPLs. The applied bias is 1.5V. b. IV curve for a thin film of HgTe/3CdS NPLs under various excitation powers ranging from dark condition to $25 \mathrm{~mW}$ from a 1.55 $\mu \mathrm{m}$ laser diode source. $c$. Responsivity as a function of light excitation power $(\lambda=1.55 \mu \mathrm{m})$ for a thin film of $\mathrm{HgTe} / 3 \mathrm{CdS}$. The applied bias is $1.5 \mathrm{~V}$, measurement is conducted at room temperature. $d$. Photocurrent as a function of time while the sample is illuminated by a pulse of $1.55 \mu \mathrm{m}$ light for different temperatures. The applied bias is 1.5V. e. Detectivity as a function of light excitation power $(\lambda=1.55 \mu \mathrm{m})$ for a thin film of $\mathrm{HgTe} / 3 \mathrm{CdS}$. The applied bias is $1.5 \mathrm{~V}$, measurement is conducted at room temperature. The inset is a measure of the noise current spectral density, under $1.5 \mathrm{~V}$.

\section{CONCLUSION}

We demonstrate the possibility to grow heterostructure from $\mathrm{Hg}$ based 2D nanoplatelets with optical absorption in the short-wave infrared. This material presents a far better-defined optical feature than the one previously for $2 \mathrm{D}$ materials based on lead chalcogenides. The material is determined to be $\mathrm{p}$-type from transport and photoemission measurements. We demonstrate that the majority carrier lifetime in confined HgTe nanocrystals is poorly depending on dimensionality but depends on confinement and is considerably extended in the case of $\mathrm{HgTe} / \mathrm{CdS}$ compared to HgTe quantum dots at the same band gap. On the other hand, the minority carrier lifetime is found to be longer in 2D NPLs compared to OD HgTe CQDs and is poorly dependent on the presence of the shell. Finally, we demonstrate that $\mathrm{HgTe} / 3 \mathrm{CdS}$ can be used for the design of fast $(<10 \mu$ s response time) short wave infrared photodetector operated at room temperature. Integration of this material into $\mathrm{nm}$ scale electrode is proposed as a path to enhance the charge dissociation and reach higher detectivity. 


\section{Experimental details}

Chemicals. Te powder (Sigma-Aldrich, $99.99 \%$ ), Cadmium oxide (CdO, Strem Chemicals, $99.99 \%$ ), Mercury acetate ( $\mathrm{Hg}(\mathrm{Ac})_{2}$, Sigma-Aldrich, > $99.0 \%$ ), Oleylamine (OAm, Acros, 80-90\%), Trioctylphosphine (TOP, Cytec, $90 \%$ ), Propionic acid (Aldrich, $99 \%)$, Cadmium bromide tetrahydrate $\left(\mathrm{CdBr}_{2},\left(\mathrm{H}_{2} \mathrm{O}\right)_{4}\right.$, Aldrich, 98\%), Ammonium sulfide solution $\left(\left(\mathrm{NH}_{4}\right)_{2} \mathrm{~S}\right.$, Fluka), Oleic acid (Aldrich, $90 \%$ ), Trioctylamine (Alfa Aesar, 95\%), Octadecene (ODE, Aldrich, $90 \%$ ), Ethanol (VWR, > $99.9 \%$ ), n-Hexane (VWR, 98 \%), Acetone (VWR, 99 \%), Methanol (Carlo Erba, > 99,9\%).

\section{Precursors}

Cadmium propionate. Cadmium propionate ( $\left.\mathrm{Cd}(\text { prop })_{2}\right)$ is obtained by mixing $1.036 \mathrm{~g}$ of $\mathrm{CdO}$ powder with $10 \mathrm{~mL}$ of propionic acid under argon flow for $1 \mathrm{~h}$ at $70{ }^{\circ} \mathrm{C}$. The flask is then open and heated at $140{ }^{\circ} \mathrm{C}$ until the volume gets reduced by a factor two. The white solution is precipitated with acetone. The solution is centrifuged and the supernatant discarded. The solid is dried under vacuum at room temperature for $24 \mathrm{~h}$.

TOPTe (1 M). In a three-neck flask, $2.54 \mathrm{~g}$ of Te powder are mixed with $20 \mathrm{~mL}$ of trioctylphosphine and degassed under vacuum at room temperature until the pressure gets reduced below $1 \mathrm{mbar}$. Then the flask is heated under argon at $275{ }^{\circ} \mathrm{C}$ until the black Te powder gets fully dissolved. The orange solution is then cooled and turns yellow. Once the temperature is below $40^{\circ} \mathrm{C}$, the solution is further degassed under vacuum for $10 \mathrm{~min}$ and finally stored in an air free glove box.

Sulfide solution. Ammonium sulfide solution (100 $\mu \mathrm{L}, 0.3 \mathrm{mmol})$, oleylamine $(125 \mu \mathrm{L}, 0.4 \mathrm{mmol})$ and $2 \mathrm{~mL}$ of hexane are introduced in a flask. $200 \mu \mathrm{L}$ of methanol are added to remove water. A biphasic mixture is obtained. To remove the excess of polysulfide, $75 \mu \mathrm{L}$ of TOP $(0.2 \mathrm{mmol})$ are introduced leading to the formation of TOPS.

\section{Nanocrystals synthesis.}

Synthesis of $3 \mathrm{MLs}$ CdTe NPLs. In a three-neck flask, Cd(prop) 2 (390 mg, $1.5 \mathrm{mmol})$, oleic acid $(240 \mu \mathrm{L}, 0,75 \mathrm{mmol})$ and $30 \mathrm{~mL}$ of octadecene are degassed under vacuum at $90^{\circ} \mathrm{C}$ for 1 hour. Under argon flow, TOPTe at $1 \mathrm{M}(300 \mu \mathrm{L})$ diluted in $1.5 \mathrm{~mL}$ of octadecene is swiftly injected at $210^{\circ} \mathrm{C}$. After 30 minutes of reaction, the mixture is cooled down and 1.5 $\mathrm{mL}$ of oleic acid is injected. The resulting nanoplatelets are precipitated twice with an excess of ethanol and are redispersed in $30 \mathrm{~mL}$ of hexane.

Cation exchange for synthesis of 3 MLs HgTe NPLs. In a flask, $110 \mathrm{~mL}$ of hexane and $2 \mathrm{~mL}$ of CdTe nanoplatelets are introduced. At room temperature, $5 \mathrm{~mL}$ of a freshly prepared solution at $0.01 \mathrm{M}$ of $\mathrm{Hg}(\mathrm{Ac})_{2}$ in trioctylamine are introduced (the ratio $\mathrm{Cd}: \mathrm{Hg}$ is 1:2). The cation exchange is followed by absorption spectroscopy. Once the first excitonic peak has reach $824 \mathrm{~nm}, 1 \mathrm{~mL}$ of oleic acid are injected to stabilize the HgTe NPLs. The excess of precursor is removed by centrifugation ( $9000 \mathrm{rpm}, 5 \mathrm{~min}$ ). The nanoplatelets are then resuspended in hexane for further experiments.

Shell growth by c-ALD. $1 \mathrm{~mL}$ of the 3 MLs HgTe NPL solution is diluted in $1 \mathrm{~mL}$ of hexane. $100 \mu \mathrm{L}$ of the sulfide solution is introduced in the NPLs solution. After a quick stirring, NPLs are precipitated by centrifugation (10 $000 \mathrm{rpm}, 30$ seconds). The nanoplatelets are redispersed in $2 \mathrm{~mL}$ of hexane. The NPLs are stable in apolar phase due to the oleylammonium on their surface. To grow the half layer of $\mathrm{Cd}, 200 \mu \mathrm{L}$ of a solution of $\mathrm{CdBr}_{2}, 4 \mathrm{H}_{2} \mathrm{O}$ in ethanol at $0.1 \mathrm{M}$ are added. The solution is vigorously stirred until the NPLs are aggregated. To obtain a stable colloidal suspension, 20 $\mu \mathrm{L}$ of oleylamine are then injected. Finally, the NPLs are precipitated and suspended in $2 \mathrm{~mL}$ of hexane. In this way, a monolayer of CdS is grown on both sides of the NPLs ( $\mathrm{HgTe} / 1 \mathrm{CdS})$. The same process is reproduced for each additional layer of CdS.

HgTe CQDs synthesis with band edge at $6000 \mathrm{~cm}^{-1}$ : $513 \mathrm{mg}$ of $\mathrm{HgCl}_{2}$ was added to $60 \mathrm{~mL}$ of oleylamine in a $100 \mathrm{~mL}$ round flask. The solution was placed under vacuum and heated to $110^{\circ} \mathrm{C}$ for $1 \mathrm{~h}$. Then, the temperature is decreased to $60{ }^{\circ} \mathrm{C}$ and solution placed to Ar atmosphere. $1.9 \mathrm{~mL}$ of TOP:Te $(1 \mathrm{M})$ with $10 \mathrm{~mL}$ of oleylamine are added to the mercury solution. The solution color gradually turns to dark brown and the reaction is made during $3 \mathrm{~min}$. A solution made of $1 \mathrm{~mL}$ of dodecanethiol and $9 \mathrm{~mL}$ of toluene is quickly added to quench the reaction. The nanocrystals are then precipitated with ethanol. After centrifugation, the nanocrystals are redispersed in chloroform. The washing step is repeated one more time. The solution is redispersed in chloroform and filtered with a $0.2 \mu \mathrm{m}$ filter. Two additional washing steps are applied with final redispersion in chloroform. 
Material characterization. For UV-Vis spectroscopy, diluted solutions of nanocrystals in hexane are prepared. The absorption spectra are acquired using a Shimadzu UV 3600 spectrometer. For electron microscopy imaging, a diluted solution of nanocrystal is dropcasted on a copper TEM grid. The grid is degassed overnight under secondary vacuum. TEM observations were performed with a JEOL 2010. For film thickness determination, we used a Dektak 150 Veeco profilometer.

Photoemission measurements. $5 \mathrm{~nm}$ of $\mathrm{Cr}$ and $80 \mathrm{~nm}$ of Au were deposited by thermal evaporation on silicon wafers to get conductive substrates. On these substrates, a drop of the HgTe-xCdS solution was deposited at room temperature and the toluene evaporated quickly. A ligand exchange with EDT was performed (see procedure in the solid-state ligand exchange section below) and the film was then introduced in the preparation chamber, degassed at room temperature until a vacuum of $10^{-10} \mathrm{mbar}$ was reached, and finally transferred in the analysis chamber. For XPS measurements, a photon energy around $600 \mathrm{eV}$ was used and the photoelectrons were detected at normal emission from the polarization vector, using a $50 \mathrm{eV}$ pass energy. The experimental value of photon energy was measured on a clean $A u$ substrate: the first and second orders of $A u 4 f_{7 / 2}$ core level were acquired and the difference of measured kinetic energies between the two orders led to actual photon energy $h v_{\text {exp }}$. Finally, to recalibrate the system, the Fermi level kinetic energy of the same clean gold substrate was acquired and shifted to be at $h v_{\exp }$ (corresponding to a binding energy of $0 \mathrm{eV}$ ). This shift was then applied to all the measured kinetic energies before calculating the binding energies using the formula $\mathrm{BE}=\mathrm{hv} \mathrm{exp}_{\mathrm{p}}-\mathrm{cKE}$ where $\mathrm{CKE}$ is the corrected kinetic energy.

\section{Device fabrication}

Electrodes fabrication. A glass substrate is first rinsed then sonicated in acetone for $5 \mathrm{~min}$. The wafer is then rinsed with acetone and isopropanol and finally $\mathrm{O}_{2}$ plasma cleaned for $5 \mathrm{~min}$. Then AZ 5214E resist is spin coated on the substrate and baked at $110^{\circ} \mathrm{C}$ for $90 \mathrm{~s}$. The resist is exposed through a shadow mask to UV illumination for $2 \mathrm{~s}$, with black paper below the substrate. The resist is then baked again at $125^{\circ} \mathrm{C}$ for $2 \mathrm{~min}$ and re-exposed to UV (without the mask) for $40 \mathrm{~s}$. The resist is then developed using AZ726. The film is then rinsed with water and finally dried. The remaining organic layer is removed by plasma cleaning. $5 \mathrm{~nm}$ of chromium and $80 \mathrm{~nm}$ of gold are evaporated and the remaining resist is removed by immersing the film in acetone for one hour. The electrodes are then rinsed with isopropanol and finally dried. The interdigitated electrodes include 25 digits, each $2.5 \mathrm{~mm}$ long with a $20 \mu \mathrm{m}$ gap between them. The optical area of the device is $0.037 \mathrm{~cm}^{2}$.

Solid state ligand exchange. Solutions at $1 \%$ in mass of EDT in ethanol are prepared. A drop of HgTe NPL solution in hexane:octane (9:1 volume ratio) is drop-casted onto the electrodes and left to dry. The resulting thin film is dipped in the ligand solution and rinsed with acetone. Once the film is dried, a second layer of HgTe NPLs is deposited and the ligand exchange step is repeated. The deposition process is performed five to six times in total.

Electrolyte gating. For electrolyte gating we first mixed in a glove box $0.5 \mathrm{~g}$ of $\mathrm{LiClO}_{4}$ with $2.3 \mathrm{~g}$ of PEG $\left(\mathrm{MW}_{\mathrm{W}}=6 \mathrm{~kg} \cdot \mathrm{mol}^{-}\right.$ $\left.{ }^{1}\right)$. The vial is heated at $170^{\circ} \mathrm{C}$ on a hot plate for $2 \mathrm{~h}$ until the solution appears clear. The electrolyte solution is warmed to around $100{ }^{\circ} \mathrm{C}$ and brushed on the top of the HgTe NPL film.

\section{SUPPORTING INFORMATIONS}

Supporting information content analysis of core level spectrum obtained from photoemission and detail about transport and phototransport characterization setup.

\section{STATEMENT OF CONTRIBUTION}

$E L$ and $\mathrm{SI}$ designed the project. EI, MD, and SI grow the material and perform material characterization. $\mathrm{CG}, \mathrm{CL}, \mathrm{BM}$, $\mathrm{NG}, \mathrm{JQ}, \mathrm{AC}, \mathrm{JR}, \mathrm{HC}, \mathrm{MS}$ and $\mathrm{EL}$ conduct the synchrotron experiment. CG, $\mathrm{HA}$, El and $\mathrm{CL}$ conduct the transport measurements. $C G$ and EL write the manuscript with inputs and feedback from all authors

\section{ACKNOWLEDEGMENTS}


EL thanks the support ERC starting grant blackQD (grant ${ }^{\circ}{ }^{\circ 56225)}$ ). We acknowledge the use of clean-room facilities from the "Centrale de Proximité Paris-Centre". This work has been supported by the Region lle-de-France in the framework of DIM Nano-K (grant dopQD). This work was supported by French state funds managed by the ANR within the Investissements d'Avenir programme under reference ANR-11-IDEX-0004-02, and more specifically within the framework of the Cluster of Excellence MATISSE and also by the grant IPER-nano2. NG and JR thank Nexdot for post doc funding and JQ thanks the Chinese scholar council for PhD grant.

\section{REFERENCES}

1 M. Nasilowski, B. Mahler, E. Lhuillier, S. Ithurria and B. Dubertret, Chem. Rev., 2016, 116, 10934-10982.

2 E. Lhuillier, S. Pedetti, S. Ithurria, B. Nadal, H. Heuclin and B. Dubertret, Acc. Chem. Res., 2015, 48, 22-30.

$3 \quad$ U.S. Patent Application, 15/561,717, 2018.

4 S. Ithurria, G. Bousquet and B. Dubertret, J. Am. Chem. Soc., 2011, 133, 3070-3077.

5 A. Riedinger, F. D. Ott, A. Mule, S. Mazzotti, P. N. Knüsel, S. J. P. Kress, F. Prins, S. C. Erwin and D. J. Norris, Nat. Mater., 2017, 16, 743-748.

6 Y. Chen, D. Chen, Z. Li and X. Peng, J. Am. Chem. Soc., 2017, 139, 10009-10019.

7 A. L. Rogach, A. Eychmüller, S. G. Hickey and S. V. Kershaw, Small, 2007, 3, 536-557.

8 S. V. Kershaw, A. S. Susha and A. L. Rogach, Chem. Soc. Rev., 2013, 42, 3033-3087.

9 C. Schliehe, B. H. Juarez, M. Pelletier, S. Jander, D. Greshnykh, M. Nagel, A. Meyer, S. Foerster, A. Kornowski, C. Klinke and H. Weller, Science (80-. )., 2010, 329, 550-553.

S. M. Premathilaka, Z. Jiang, A. Antu, J. Leffler, J. Hu, A. Roy and L. Sun, Phys. Status Solidi - Rapid Res. Lett., 2016, 10, 838-842.

11 H. Zhang, B. H. Savitzky, J. Yang, J. T. Newman, K. A. Perez, B. R. Hyun, L. F. Kourkoutis, T. Hanrath and F. W. Wise, Chem. Mater., 2016, 28, 127-134.

12 A. H. Khan, S. Pal, A. Dalui, J. Pradhan, D. D. Sarma and S. Acharya, Chem. Mater., 2017, 29, 1175-1182.

13 S. Khan, Z. Jiang, S. M. Premathilka, A. Antu, J. Hu, A. A. Voevodin, P. J. Roland, R. J. Ellingson and L. Sun, Chem. Mater., 2016, 28, 5342-5346.

14 G. B. Bhandari, K. Subedi, Y. He, Z. Jiang, M. Leopold, N. Reilly, H. P. Lu, A. T. Zayak and L. Sun, Chem. Mater., 2014, 26, 5433-5436.

M. M. Ramin Moayed, T. Bielewicz, H. Noei, A. Stierle and C. Klinke, Adv. Funct. Mater., 2018, 28, 1706815.

T. Bielewicz, M. M. Ramin Moayed, V. Lebedeva, C. Strelow, A. Rieckmann and C. Klinke, Chem. Mater., 2015, 27, 8248-8254.

A. H. Khan, R. Brescia, A. Polovitsyn, I. Angeloni, B. Martín-García and I. Moreels, Chem. Mater., 2017, 29, $2883-2889$.

T. Bielewicz, S. Dogan and C. Klinke, Small, 2015, 11, 826-833.

S. Dogan, T. Bielewicz, Y. Cai and C. Klinke, Appl. Phys. Lett., 2012, 101, 073102.

E. Izquierdo, A. Robin, S. Keuleyan, N. Lequeux, E. Lhuillier and S. Ithurria, J. Am. Chem. Soc., 2016, 138, 10496-10501. 
A. M. Smith and S. Nie, J. Am. Chem. Soc., 2011, 133, 24-26.

E. Izquierdo, M. Dufour, A. Chu, C. Livache, B. Martinez, D. Amelot, G. Patriarche, N. Lequeux, E. Lhuillier and S. Ithurria, Chem. Mater., 2018, 30, 4065-4072.

A. W. Achtstein, A. Schliwa, A. Prudnikau, M. Hardzei, M. V. Artemyev, C. Thomsen and U. Woggon, Nano Lett., 2012, 12, 3151-3157.

R. Benchamekh, N. A. Gippius, J. Even, M. O. Nestoklon, J. M. Jancu, S. Ithurria, B. Dubertret, A. L. Efros and P. Voisin, Phys. Rev. B - Condens. Matter Mater. Phys., 2014, 89, 035307.

E. Lhuillier, J. F. Dayen, D. O. Thomas, A. Robin, B. Doudin and B. Dubertret, Nano Lett., 2015, 15, 1736-1742.

A. Robin, E. Lhuillier, X. Z. Xu, S. Ithurria, H. Aubin, A. Ouerghi and B. Dubertret, Sci. Rep., 2016, 6, 24909.

S. Pedetti, B. Nadal, E. Lhuillier, B. Mahler, C. Bouet, B. Abécassis, X. Xu and B. Dubertret, Chem. Mater., 2013, 25, 2455-2462.

S. Ithurria and D. V. Talapin, J. Am. Chem. Soc., 2012, 134, 18585-18590.

A. Antanovich, A. W. Achtstein, A. Matsukovich, A. Prudnikau, P. Bhaskar, V. Gurin, M. Molinari and M. Artemyev, Nanoscale, 2017, 9, 18042-18053.

A. M. Jagtap, B. Martinez, N. Goubet, A. Chu, C. Livache, C. Greboval, julien Ramade, D. Amelot, P. trousset, A. Triboulin, S. Ithurria, M. G. Silly, B. Dubertret and E. Lhuillier, ACS Photonics, 2018, acsphotonics.8b01032.

A. H. Nethercot, Phys. Rev. Lett., 1974, 33, 1088-1091.

E. Lhuillier, S. Ithurria, A. Descamps-Mandine, T. Douillard, R. Castaing, X. Z. Xu, P. L. Taberna, P. Simon, H. Aubin and B. Dubertret, J. Phys. Chem. C, 2015, 119, 21795-21799.

B. F. Spencer, D. M. Graham, S. J. O. Hardman, E. A. Seddon, M. J. Cliffe, K. L. Syres, A. G. Thomas, S. K. Stubbs, F. Sirotti, M. G. Silly, P. F. Kirkham, A. R. Kumarasinghe, G. J. Hirst, A. J. Moss, S. F. Hill, D. A. Shaw, S. Chattopadhyay and W. R. Flavell, Phys. Rev. B - Condens. Matter Mater. Phys., 2013, 88, 195301.

B. F. Spencer, M. J. Cliffe, D. M. Graham, S. J. O. Hardman, E. A. Seddon, K. L. Syres, A. G. Thomas, F. Sirotti, M. G. Silly, J. Akhtar, P. O’Brien, S. M. Fairclough, J. M. Smith, S. Chattopadhyay and W. R. Flavell, Surf. Sci., 2015, 641, 320-325.

C. Livache, E. Izquierdo, B. Martinez, M. Dufour, D. Pierucci, S. Keuleyan, H. Cruguel, L. Becerra, J. L. Fave, H. Aubin, A. Ouerghi, E. Lacaze, M. G. Silly, B. Dubertret, S. Ithurria and E. Lhuillier, Nano Lett., 2017, 17, 40674074.

B. Martinez, C. Livache, N. Goubet, A. Jagtap, H. Cruguel, A. Ouerghi, E. Lacaze, M. G. Silly and E. Lhuillier, J. Phys. Chem. C, 2018, 122, 859-865.

W. J. Mir, C. Livache, N. Goubet, B. Martinez, A. Jagtap, A. Chu, N. Coutard, H. Cruguel, T. Barisien, S. Ithurria, A. Nag, B. Dubertret, A. Ouerghi, M. G. Silly and E. Lhuillier, Appl. Phys. Lett., 2018, 112, 113503.

S. Keuleyan, J. Kohler and P. Guyot-Sionnest, J. Phys. Chem. C, 2014, 118, 2749-2753.

J. Orenstein, M. A. Kastner and V. Vaninov, Philos. Mag. B Phys. Condens. Matter; Stat. Mech. Electron. Opt. Magn. Prop., 1982, 46, 23-62.

J. Orenstein and M. Kastner, Phys. Rev. Lett., 1981, 46, 1421-1424. 

Bodnarchuk, M. V. Kovalenko and W. Heiss, ACS Nano, 2014, 8, 12883-12894.

47 D. D. W. Grinolds, P. R. Brown, D. K. Harris, V. Bulovic and M. G. Bawendi, Nano Lett., 2015, 15, 21-26.

48 G. Allan and C. Delerue, Phys. Rev. B - Condens. Matter Mater. Phys., 2012, 86, 165437. 